\title{
Durable Response to Vemurafenib and Cobimetinib for the Treatment of BRAF-Mutated Metastatic Melanoma in Routine Clinical Practice
}

\author{
$M^{a}$ del Carmen Álamo' \\ Sebastian Ochenduszko ${ }^{2}$ \\ Guillermo Crespo ${ }^{3}$ \\ Mónica Corral (iD) \\ Juana Oramas ${ }^{5}$ \\ Pilar Sancho ${ }^{6}$ \\ Javier Medina ${ }^{7}$ \\ Fernando Garicano ${ }^{8}$ \\ Pedro López ${ }^{9}$ \\ Begoña Campos Balea $\mathbb{D}^{10}$ \\ Analia Rodríguez Garzotto" \\ Eva Muñoz-Couselo' ${ }^{12,13}$ \\ 'Oncology Department, Hospital \\ Universitario Virgen Macarena, Sevilla, Spain; \\ ${ }^{2}$ Oncology Department, Hospital \\ Universitario Dr. Peset, Valencia, Spain; \\ ${ }^{3}$ Oncology Department, Hospital \\ Universitario de Burgos, Burgos, Spain; \\ ${ }^{4}$ Oncology Department, Hospital Clínico \\ Universitario Lozano Blesa, Zaragoza, Spain; \\ ${ }^{5}$ Oncology Department, Hospital \\ Universitario de Canarias, Santa Cruz de \\ Tenerife, Spain; ${ }^{6}$ Oncology Department, \\ Hospital Universitario Virgen del Rocío, \\ Sevilla, Spain; ${ }^{7}$ Oncology Department, \\ Hospital Universitario Virgen de la Salud, \\ Toledo, Spain; ${ }^{8}$ Oncology Department, \\ Hospital de Galdakao, Bizkaia, Spain; \\ ${ }^{9}$ Oncology Department, Complejo \\ Hospitalario General de Jaén, Jaén, Spain; \\ ${ }^{10}$ Oncology Department, Hospital Lucus \\ Augusti, Lugo, Spain; '"Medical Department \\ and Strategy, Roche S.A, Madrid, Spain; \\ ${ }^{12}$ Oncology Department, Hospital \\ Universitario Vall d'Hebron, Barcelona, \\ Spain; ${ }^{13} \mathrm{VHIO}$ Vall d'Hebron Institute on \\ Oncology, Barcelona, Spain
}

Correspondence: Eva Muñoz-Couselo Oncology Department, Hospital

Universitario Vall d'Hebron, Passeig de la

Vall d'Hebron, I I9, Barcelona, 08035, Spain

Email emunoz@vhio.net
Background: The combination of $B R A F$ and $M E K$ inhibitors delays the onset of resistance and provides more sustained and dramatic responses in comparison with a $B R A F$ inhibitor in monotherapy. The objective of the study was to evaluate the effectiveness of the combination therapy with vemurafenib/cobimetinib in terms of durability, and to describe differential characteristics in patients associated to durable responses in real-world settings.

Patients and Methods: Retrospective, observational, cross-sectional, multicenter study involving 41 patients with advanced melanoma harboring a $B R A F^{\mathrm{V} 600}$ mutation who initiated a combination therapy with vemurafenib/cobimetinib between May 2018 and March 2019 . Participants were differentiated regarding the durability of the response: durable (complete response, $\mathrm{CR}$, or a partial response, $\mathrm{PR}$, for at least 12 months) and non-durable (stable disease, $\mathrm{SD}$, progressive disease, $\mathrm{PD}$, or $\mathrm{CR} / \mathrm{PR}<12$ months). Secondary endpoints included treatment adherence, labor productivity, anxiety/depression, and safety profile.

Results: During the combination therapy, 12 patients $(29.3 \%)$ had a CR, 19 a PR (46.3\%), 5 showed SD (12.2\%), and 5 had PD. A total of 12 patients $(29.3 \%)$ were considered as achieving a durable response and $29(70.7 \%)$ as a non-durable one. Practically all sociodemographic and clinical characteristics were similar between patients. Body mass index was the only differential factor (with higher body mass index achieving a non-durable response). The treatment adherence was $100 \%$ in patients with durable response and $66.7 \%$ in those with non-durable.

Conclusion: The combination treatment with vemurafenib/cobimetinib results in an important impact on long-term survival, leading to a steady CR in one-third of the patients.

Keywords: vemurafenib, cobimetinib, BRAF, metastatic melanoma, durable response, clinical practice

\section{Introduction}

Melanoma represents a substantial and growing public health burden. ${ }^{1}$ According to the World Health Organization, 287,723 new cases of melanoma were reported in 2018, with 60,712 deaths. $^{2}$ The worldwide incidence is 3.5 and 4.0 per 100,000 inhabitants among men and women, respectively. Most of cases (83\%) are diagnosed at a localized stage, showing a 5-year survival of $99.0 \%{ }^{3}$ However, when spread to regional lymph nodes ( $9 \%$ of newly diagnoses), 5-year survival decreases to $66.2 \%$, and when metastasizes (4\% of cases) it reduces to $27.3 \%$. Between $40-$ $60 \%$ of cutaneous melanomas harbor a mutation in $B R A F$ gene, predominantly $\left(\geq 97 \%\right.$ ) in the codon $600 .^{4}$ The most frequent mutation $(90 \%)$ consists of the 
substitution of valine for glutamic acid $\left(B R A F^{\mathrm{V} 600 \mathrm{E}}\right)$, followed by the substitution for lysine (BRAF $\mathrm{V} 600 \mathrm{~K}$, between $8-20 \%$ of patients), arginine (BRAF $\left.{ }^{\mathrm{V} 600 \mathrm{R}}, 1 \%\right)$, methionine $\left(B R A F^{\mathrm{V} 600 \mathrm{M}}, 0.3 \%\right)$, and aspartic acid $(B R A F$ V600D, $0.1 \%)$. BRAF mutant melanomas are associated with more aggressive biological behaviors and reduced survival than wild-type ones. ${ }^{5,6}$ The discovery of $B R A F$ mutations led to the development of targeted therapies, including selective inhibitors of the $B R A F^{\mathrm{V} 600}$-mutated kinase (vemurafenib, dabrafenib and encorafenib) and inhibitors of the downstream $M E K$ kinase (trametinib, cobimetinib, and binimetinib). ${ }^{4}$ Vemurafenib was the first approved $B R A F$ inhibitor, based on results from the Phase III BRIM-3 trial which demonstrated significant improvement with vemurafenib in overall survival (OS, 13.6 months) and progression-free survival (PFS, 5.3 months) compared with dacarbazine (9.7 and 1.6 months, respectively) for metastatic $B R A F$ mutant melanoma. ${ }^{7}$ Despite the proven clinical benefit of $B R A F$ inhibitors, only $5 \%$ of patients achieve a complete response $(\mathrm{CR})$, and the disease frequently progresses approximately 6-7 months after initiating the treatment due to acquired resistance. ${ }^{8}$ The reactivation of the mitogen-activated protein kinase pathway represents the main cause of resistance to $B R A F$ inhibitors. This resistance affects directly to the rate and duration of tumor responses. ${ }^{8}$ There is thus a clinical need to identify the most effective therapy for these patients. The combination therapy of a $B R A F$ and $M E K$ inhibitor (such as vemurafenib/cobimetinib) has been shown to improve substantially survival, and to provide more durable and greater tumor responses than $B R A F$ monotherapy in patients with $B R A F^{\mathrm{V} 600}$ mutant advanced melanoma. ${ }^{7}$ The 5-year follow-up data of the double-blind, randomized, multicenter, phase III coBRIM trial, involving 495 patients with $B R A F^{\mathrm{V} 600}$ mutant melanoma, has recently revealed a superior median PFS with vemurafenib/cobimetinib (12.6 months) and objective response rate (70\%) than vemurafenib plus placebo (7.2 months and $50 \%$, respectively). ${ }^{9}$ Among patients receiving vemurafenib/cobimetinib, median OS and PFS were higher in patients with normal lactate dehydrogenase (LDH) at baseline (38.5 and 15.0 months, $n=131)$ than with elevated LDH (14.8 and 8.6 months, $n=112)$. Furthermore, coBRIM trial originally showed that the health-related quality of life of patients was maintained with vemurafenib/cobimetinib, in contrast to vemurafenib/placebo. ${ }^{10}$ To date, there are no published studies specifically designed to address the effect of vemurafenib/cobimetinib in routine clinical practice, or to determine which patients can achieve long-term clinical effects. Therefore, the objective of the present study was to evaluate the effectiveness of the combination therapy in terms of durability, and to describe differential characteristics of patients associated with durable responses in real-world settings.

\section{Patients and Methods Study Design}

Retrospective, observational, cross-sectional, multicenter study involving patients with advanced melanoma harboring a $B R A F^{\mathrm{V} 600}$ mutation who initiated a combination therapy with vemurafenib/cobimetinib between May 2018 and March 2019. A total of 15 centers across Spain participated in the study. Exclusion criteria were: having received any prior treatment for melanoma in the metastatic setting; lack of medical records from the last 12 months (before study inclusion); participation in another clinical study; or having a mental disease or being unable to make decisions and follow instructions.

\section{Endpoints and Study Variables}

The primary endpoint included the percentage of patients with durable clinical response to the combination therapy with $B R A F$ and $M E K$ inhibitors, and the comparison of sociodemographic and clinical characteristics of patients with durable and non-durable responses. A patient was considered to achieve a durable clinical response when having a $\mathrm{CR}$ or a partial response (PR) for at least 12 months. In case of stable disease (SD), progressive disease (PD), or CR/PR for less than 12 months, the patient was considered to achieve a non-durable clinical response. Evaluated sociodemographic and clinical characteristics of patients were age, gender, race, body mass index (BMI), Eastern Cooperative Oncology Group performance status at diagnosis, primary tumor location, type of $B R A F$ mutation, tumor stage at treatment initiation, and metastatic location at treatment initiation. Other effectiveness variables included: time to response, duration of the response, and time to progression. The time to response was defined as the time between the initiation of the combination treatment and the best response achieved. The duration of the response was defined as the time elapsed between the best response achieved and the progression or death, by any cause. The time to progression was defined as the time elapsed between the start of the treatment and progression. Tumor stage at treatment 
initiation was determined with the 2009 version of the American Joint Committee on Cancer melanoma staging and classification. ${ }^{11}$ Additionally, secondary endpoints included the determination of the treatment adherence, labor productivity, anxiety/depression, and the safety profile. Adherence to the treatment was determined by using the 6-item simplified medication adherence questionnaire (SMAQ) in patients with active treatment at the time of the study visit. $^{12}$ A negative result in the SMAQ Questionnaire represents adherence to the treatment, whereas a positive result stands for non-adherence. Labor productivity was determined with the work productivity and activity impairment questionnaire (WPAI-GH), in patients with active treatment at the time of the study. ${ }^{13}$ Only the item of "activity impairment" (percent activity impairment due to health) was determined. Anxiety and depression were measured using the Hospital Anxiety and Depression Scale (HADS), in patients with active treatment at the time of the study visit. ${ }^{14}$ The HADS describes 14 items and global (range score: 0-42) and dimensions score (anxiety and depression, range score: 0-21 each one). Scores of dimensions were categorized into: normal (score 0-7), borderline abnormal (score 8-10) and abnormal (score 11-21). The analysis of safety outcomes was based on the incidence and severity of adverse events (AEs), using the Common Terminology Criteria for Adverse Events v4.0, and summarizing according to the system organ class, with MedDRA 21.0.

\section{Sample Size Determination and Statistical Analysis}

The sample size was determined according to the primary objective, ie percentage of patients with a durable clinical response and the description of differential characteristics associated with the response. It was estimated that 53 patients could provide a precision of $\pm 13.5 \%$ in the proportion of patient's characteristics; with a 0.95 of confidence level. Assuming 5\% of patients with non-evaluable data, the estimated sample size was 55-56 patients. Continuous variables were expressed as mean, standard deviation (SD), median, or interquartile range (25th-75th percentile), whereas categorical ones as absolute and relative frequencies. Comparisons of sociodemographic and clinical characteristics of patients were performed using the Mann-Whitney $U$-test, in continuous variables, and chi-square or Fisher's exact tests, in categorical ones, when appropriate. Statistical significance was established when $\mathrm{P} \leq 0.05$. All statistical procedures were carried out with SAS 9.4.

\section{Results}

A total of 48 patients were initially recruited; however, only 41 were evaluable for the primary objective. During the combination therapy, 12 patients (out of 41, 29.3\%) had a CR, 19 patients a PR (46.3\%), 5 patients showed SD (12.2\%), and 5 patients had PD (12.2\%; Table 1). Median time to the best response was: 5.0 months (IQR, 2.9-7.2 months) for CR; 2.1 months (IQR, 1.8-3.4 months) for PR; and 2.2 months (IQR, 2.0-2.8 months) for SD. The median duration of the response was 7.3 months (IQR, 6.8-12.1 months) in patients with $\mathrm{CR}$, and 4.8 months (IQR, 1.0-16.0 months) and 7.3 months (IQR, 7.0-7.4 months) for those with $\mathrm{PR}$ and $\mathrm{SD}$, respectively. In patients with $\mathrm{PD}$, the median time to progression was 3.5 months (IQR, 2.6-5.5 months).

According to the durability of the clinical response, 12 patients (29.3\%) were considered as achieving a durable response, and $29(70.7 \%)$ as a non-durable one. In the group of patients with non-durable response, $9(31.0 \%$ of them) and 10 patients $(34.5 \%)$ had a CR and PR lasting less than 12 months, respectively; 5 patients (17.2\%) had SD, and 5 showed PD (17.2\%). The median duration of the response in patients with a non-durable response was 5.4 months (IQR, 2.1-7.4 months). Sociodemographic and clinical characteristics in patients

Table I Best Response Achieved During the Combination Therapy in Total Patients

\begin{tabular}{|l|c|}
\hline & Value \\
\hline Best response achieved, $\mathrm{n}(\%)$ & \\
Complete response & $12(29.3)$ \\
Partial response & $19(46.3)$ \\
Stable disease & $5(12.2)$ \\
Progressive disease & $5(12.2)$ \\
\hline Time to response, median months (IQR) & \\
Complete response & $5.0(2.9-7.2)$ \\
Partial response & $2.1(1.8-3.4)$ \\
Stable disease & $2.2(2.0-2.8)$ \\
\hline Duration of the response, median months (IQR) & \\
Complete response & $7.3(6.8-12.1)$ \\
Partial response & $4.8(1.0-16.0)$ \\
Stable disease & $7.3(7.0-7.4)$ \\
\hline Time to progression, median months (IQR) & $3.5(2.6-5.5)$ \\
\hline
\end{tabular}

Abbreviation: IQR, interquartile range. 
Table 2 Sociodemographic and Clinical Characteristics in Patients Considering the Durability of the Response

\begin{tabular}{|c|c|c|c|}
\hline & $\begin{array}{c}\text { Patients } \\
\text { with } \\
\text { Durable } \\
\text { Clinical } \\
\text { Response } \\
(\mathrm{N}=12)\end{array}$ & $\begin{array}{c}\text { Patients } \\
\text { with Non- } \\
\text { Durable } \\
\text { Clinical } \\
\text { Response } \\
(\mathbf{N}=29)\end{array}$ & $P$ value \\
\hline Age, mean years (SD) & $55.8(16.5)$ & $58.5(13.1)$ & 0.716 \\
\hline $\begin{array}{l}\text { Gender, } \mathrm{n}(\%) \\
\quad \text { Male } \\
\text { Female }\end{array}$ & $\begin{array}{l}4(33.3) \\
8(66.7)\end{array}$ & $\begin{array}{l}19(65.5) \\
10(34.5)\end{array}$ & 0.087 \\
\hline $\begin{array}{l}\text { Race, n (\%) } \\
\text { Caucasian } \\
\text { Black }\end{array}$ & $\begin{array}{c}12(100.0) \\
0(0.0)\end{array}$ & $\begin{array}{c}28(96.6) \\
I(3.4)\end{array}$ & 1.000 \\
\hline BMI, mean $\mathrm{Kg} / \mathrm{m}^{2}$ (SD) & $24.4(6.0)$ & $29.2(5.6)$ & 0.028 \\
\hline $\begin{array}{l}\text { ECOG PS at diagnosis, } n(\%) \\
0 \\
1 \\
2 \\
3\end{array}$ & $\begin{array}{l}\text { II }(91.7 \%) \\
\text { I (8.3) } \\
0(0.0) \\
0(0.0)\end{array}$ & $\begin{array}{c}23(79.3) \\
4(13.8) \\
1(3.4) \\
1(3.4)\end{array}$ & 1.000 \\
\hline $\begin{array}{l}\text { Primary tumor location, n (\%) } \\
\text { Skin } \\
\text { Cervix } \\
\text { Data not available }\end{array}$ & $\begin{array}{l}\text { II }(91.7) \\
\text { I (8.3) } \\
0(0.0)\end{array}$ & $\begin{array}{c}22(75.9) \\
0(0.0) \\
7(24.1)\end{array}$ & 0.059 \\
\hline $\begin{array}{l}\text { Type of BRAF mutation, } \mathrm{n}(\%) \\
\text { V600E } \\
\text { Unspecific }\end{array}$ & $\begin{array}{l}6(50.0) \\
6(50.0)\end{array}$ & $\begin{array}{c}20(69.0) \\
9(31.0)\end{array}$ & 0.251 \\
\hline $\begin{array}{l}\text { Tumor stage at treatment } \\
\text { initiation, } \mathrm{n}(\%) \\
\text { IIlc unresectable } \\
\text { IV }\end{array}$ & $\begin{array}{c}\text { I (8.3) } \\
\text { II (9I.7) }\end{array}$ & $\begin{array}{c}3(10.3) \\
26(89.7)\end{array}$ & 1.000 \\
\hline \multicolumn{4}{|l|}{$\begin{array}{l}\text { Metastatic location at treatment } \\
\text { initiation, } \mathrm{n}(\%)\end{array}$} \\
\hline Lung & $6(50.0)$ & $15(51.7)$ & 1.000 \\
\hline Liver & $3(25.0)$ & $7(24.1)$ & 1.000 \\
\hline Skin & $2(16.7)$ & $8(27.6)$ & 0.694 \\
\hline Brain & $0(0.0)$ & $2(6.9)$ & 1.000 \\
\hline Other & $7(58.3)$ & $19(65.5)$ & 0.730 \\
\hline
\end{tabular}

Abbreviations: BMI, body mass index; ECOG PS, Eastern Cooperative Oncology Group performance status.

considering the durability of the response are shown in Table 2. With the exception of BMI (mean, $24.4 \mathrm{Kg} / \mathrm{m}^{2}$, $\mathrm{SD}$, 6.0 with durable response versus $29.2 \mathrm{Kg} / \mathrm{m}^{2}$, SD 5.6, with non-durable response; $\mathrm{P}=0.028$ ), all sociodemographic and clinical characteristics of patients were similar among groups. The mean age of patients was 57.8 years (SD, 14.0 years). A slightly higher percentage of patients with durable response was female $(66.7 \%)$, in contrast to the non-durable response $(34.5 \%)$. The primary tumor was predominantly located in the skin (numerically higher in the group with durable response, $91.7 \%$, versus non-durable, $75.9 \%$ ). Main location of metastases were: lung $(50.0 \%$ versus $51.7 \%)$, liver ( $25.0 \%$ versus $24.1 \%)$, and skin $(16.7 \%$ versus $27.6 \%)$. Treatment characteristics in total patients considering the durability of the response are shown in Table 3. The adherence to the treatment was $100 \%$ in patients with durable response and $66.7 \%$ with non-durable one. Regarding labor productivity, no differences were found in activity impairment between patients with durable response (mean, 35.0; SD, 17.3) and non-durable response (mean, 23.3; SD, 40.4). One patient (out of 4, $25.0 \%$ ) and 2 patients (out of 3,66.7\%) from the group with and without durable response, respectively, showed an abnormal response in the HADS anxiety scale. Considering depression, one patient from the group with non-durable response showed an abnormal response in the scale. No statistical differences were found between groups in anxiety and depression symptoms.

Regarding safety, 261 AEs (40.5\% of total) were reported by patients with durable response, and 384 AEs $(59.5 \%)$ by those with non-durable response. A total of 18 AEs (2.8\%) were considered as serious (4 in group with durable response and 14 without it). Of AEs, $45.9 \%$ of cases were related with cobimetinib, 51.3\% with vemurafenib, and $42.5 \%$ with both cobimetinib and vemurafenib (the relationship was not available in approximately $9 \%$ of AEs). Most frequent AEs related to the combined therapy considering the durability of the response are shown in Table 4. Most frequent AEs were: asthenia $(58.3 \%$ of patients with durable clinical response versus $37.9 \%$ with non-durable one), diarrhea (41.7 versus $27.6 \%$ ), arthralgia (50.0 versus $10.3 \%)$, erythema and rash (25.0 versus $17.2 \%$, in each), and photosensitivity reaction (16.7 versus $17.2 \%$ ). Severity was mild in $68.5 \%$ of AEs, and moderate in $19.4 \%$. In $74.9 \%$ of cases no action was required. Of AEs, $83.4 \%$ were resolved.

\section{Discussion}

Great effort has been made to identify novel therapeutic agents that aim specific targets in melanoma. ${ }^{15} B R A F$ inhibitors have demonstrated greater clinical efficacy than conventional chemotherapy for the treatment of $B R A F$ mutant melanomas. ${ }^{4,7}$ Nevertheless, they are also associated with limited objective responses and the development of resistance. ${ }^{8}$ The combination of BRAF and 
Table 3 Treatment Characteristics in Total Patients Considering the Durability of the Response

\begin{tabular}{|c|c|c|c|}
\hline & $\begin{array}{l}\text { Patients with Durable Clinical } \\
\text { Response }(\mathrm{N}=\mid 2)\end{array}$ & $\begin{array}{l}\text { Patients with Non-Durable Clinical } \\
\text { Response }(\mathrm{N}=\mathbf{2 9})\end{array}$ & $P$ value \\
\hline Dose modification, $\mathrm{n}$ (\%) & $8(66.7)$ & $15(51.7)$ & 0.497 \\
\hline \multicolumn{4}{|l|}{ Reasons * } \\
\hline Toxicity & $12(85.7)$ & $24(80.0)$ & 1.000 \\
\hline Investigator decision & $0(0.0)$ & $4(12.3)$ & 0.290 \\
\hline Clinical reasons & $2(14.3)$ & $2(6.7)$ & 0.581 \\
\hline $\begin{array}{l}\text { Time until dose modification, } \\
\text { median months (IQR) }\end{array}$ & $6.5(2.5-11.8)$ & $1.8(0.5-5.3)$ & 0.129 \\
\hline \multicolumn{4}{|l|}{ Adherence to treatment, $\mathrm{n}(\%) * *$} \\
\hline Adherent & $4(100.0)$ & $2(66.7)$ & 0.429 \\
\hline Non-adherent & $0(0.0)$ & I (33.3) & \\
\hline Concurrent radiation therapy, $\mathrm{n}(\%)$ & & & 1.000 \\
\hline Yes & I (8.3) & $3(10.3)$ & \\
\hline No & II (9I.7) & $26(89.7)$ & \\
\hline Oncologic surgery, n (\%) & & & 1.000 \\
\hline Yes & $0(0.0)$ & $2(6.9)$ & \\
\hline No & $12(100.0)$ & $27(93.1)$ & \\
\hline
\end{tabular}

Notes: *The percentage in each group was calculated according to the total number of modifications ( 14 and 30 in patients with and without a long-term clinical response, respectively). **Adherence calculated over patients with active treatment at the moment of the study visit ( 4 and 3 in patients with and without a long-term clinical response, respectively).

Abbreviation: IQR, interquartile range.

MEK inhibitors has been proven to delay the onset of resistance and provide more sustained and dramatic responses in comparison with a BRAF inhibitor in monotherapy. Franken et al, ${ }^{16}$ in a systematic literature review and network meta-analysis, identified the combination of vemurafenib/cobimetinib as one of the most favorable treatments for advanced melanoma in terms of PFS (hazard ratio, HR, 0.2). The majority of information on

Table 4 Most Frequent Adverse Events Related to the Combined Therapy in Patients Considering the Durability of the Response

\begin{tabular}{|l|c|c|}
\hline n (\%) & $\begin{array}{c}\text { Patients with } \\
\text { Durable Clinical } \\
\text { Response (N=12) }\end{array}$ & $\begin{array}{c}\text { Patients with Non- } \\
\text { Durable Clinical } \\
\text { Response (N=29) }\end{array}$ \\
\hline Asthenia & $7(58.3)$ & $11(37.9)$ \\
Diarrhea & $5(41.7)$ & $8(27.6)$ \\
Arthralgia & $6(50.0)$ & $3(10.3)$ \\
Erythema & $3(25.0)$ & $5(17.2)$ \\
Rash & $3(25.0)$ & $5(17.2)$ \\
Photosensitivity reaction & $2(16.7)$ & $5(17.2)$ \\
Skin toxicity & $3(25.0)$ & $3(10.3)$ \\
Nausea & $4(33.3)$ & $2(6.9)$ \\
Pruritus & $2(16.7)$ & $2(6.9)$ \\
Anemia & $2(16.7)$ & $2(6.9)$ \\
\hline
\end{tabular}

the efficacy of vemurafenib/cobimetinib derives from clinical trials. ${ }^{9}$ To our knowledge, none of the studies have reported the efficacy of this combination of agents in realworld settings. Only an abstract by Guardo et al, ${ }^{17}$ involving 14 patients with $B R A F$ mutant metastatic melanoma, reported the experience with vemurafenib/cobimetinib from an Italian Center. The median follow-up was 23 weeks. A total of 3 patients achieved a CR, 8 a PR, 1 $\mathrm{SD}$, and $2 \mathrm{PD}$. The objective response rate was $78.5 \%$ (11 cases). Most frequent AEs were: grade 1-2 rash (4 patients), grade 1 aspartate/alanine aminotransferase elevation ( 1 patient), grade 2 total bilirubin increase (1 patient), and grade 1 diarrhea (1 patient). Other real-world studies have evaluated the efficacy of $B R A F$ and $M E K$ inhibitors in monotherapy or in combination, but including predominantly patients receiving encorafenib/binimetinib and/or dabrafenib/trametinib; being thus non comparable with our results. ${ }^{18-21}$ In our present study, $29.3 \%$ of patients achieved a durable response of at least 12 months. The onset of the response was 6.9 months in patients having a CR, and 3.3 months in those with a PR. Given the proven efficacy of therapies, it has become necessary to identify patients who may benefit from each type of agent. $^{22}$ In our study, practically all sociodemographic 
and clinical characteristics were similar between patients with durable and non-durable clinical responses. The BMI was the only differential factor (patients with higher BMI achieving a non-durable response). Although obesity is a known risk factor for the development of cancer and poor prognosis, ${ }^{23}$ and our observation would be in line with it, diverse studies have associated overweight and early obese states with improved survival; what has been called "the obesity paradox". ${ }^{24}$ Indeed, McQuade et al, ${ }^{25}$ in a retrospective, multicohort analysis with 2046 patients with metastatic melanoma who had received $B R A F$ and $M E K$ targeted therapy, immunotherapy, or chemotherapy, concluded that obesity was correlated with improved PFS (HR: 0.8 ; 95\% confidence interval, 95\% CI: $0.7-0.9$ ) and OS (HR: 0.7; 95\% CI: 0.6-1.0), in comparison with normal BMI. Yet, other studies have demonstrated opposite results. ${ }^{26,27}$ Fang et al, ${ }^{27}$ in a study involving 1186 patients with melanoma showed significant associations between higher BMI and poorer survival outcomes (HR for OS was 1.2; 95\% CI: 1.1-1.3), after adjustment for sex, age, and stage. In our study, treatment adherence was also similar among patients with durable and non-durable clinical response. Therefore, durability of the response seems not to be associated with adherence to the treatment. Nevertheless, given the low number of patients with data on treatment adherence, no strong conclusions can be made in this regard.

On the other side, safety profile of the $B R A F / M E K$ combination therapy has not been associated with an increase in the incidence of AEs, in comparison with agents in monotherapy. ${ }^{28}$ In our study, even though the AEs caused by the combination treatment led to dose modification in $56.1 \%$ of cases, most of patients $(85.7 \%)$ were adherent to the treatment. This fact was also reinforced by the fact that patients could experience anxiety ( $42.9 \%$ of total) or depression symptoms $(14.3 \%)$. The main limitation of our study was its retrospective design, providing only available information. Another limitation was the low number of patients included in the study; however it derives directly from the availability of this subpopulation of patients in real-world settings. Furthermore, it cannot be strongly concluded that BMI is a factor associated with durable or non-durable responses, as the study was not specifically designed to identify factors associated with durable clinical responses. Besides these limitations, results are in agreement with observed previously in clinical trials. ${ }^{9}$

In conclusion, the combination treatment with vemurafenib/cobimetinib results in an important impact on long- term survival, leading to a steady $\mathrm{CR}$ in one third of the patients. Further prospective study, involving larger cohort of patients, are needed to corroborate these results.

\section{Data Sharing Statement}

Qualified researchers may request access to individual patient level data through the clinical study data request platform (https://vivli.org/). Further details on Roche's criteria for eligible studies are available here (https:// vivli.org/members/ourmembers/). For further details on Roche's Global Policy on the Sharing of Clinical Information and how to request access to related clinical study documents, see here (https://www.roche.com/ research and development/who we are how we work/ clinical_trials/our_commitment_to_data_sharing.htm).

\section{Ethical Approval and Consent Statement}

Procedures were approved by the Ethics Committee of the Hospital Vall d'Hebron, and in concordance with the Declaration of Helsinki. Excepting deceased patients (whose informed consent was waived by the Hospital Vall d'Ebron), all subjects signed an informed consent to be included in the study. In any case, patient data confidentiality was guaranteed.

\section{Acknowledgments}

The authors of this study would like to thank ADKNOMA for the editorial support.

\section{Author Contributions}

All authors made a significant contribution to the work reported, whether that is in the conception, study design, execution, acquisition of data, analysis and interpretation, or in all these areas; took part in drafting, revising or critically reviewing the article; gave final approval of the version to be published; have agreed on the journal to which the article has been submitted; and agree to be accountable for all aspects of the work.

\section{Funding}

This study was sponsored and funded by Roche Farma S. A. Spain.

\section{Disclosure}

$\mathrm{BCB}$ declares that has received consulting honoraria from Boehringer and Sanofi and is on the speaker's bureau of 
Roche, Novartis, Merck Sharp \& Dohme, Bristol-Myers Squibb, Rovi, Leopharma, Astra Zeneca, Leo-Pharma, and Sanofi. EMC declares that has received consulting honoraria, including travel and accommodation paid, and is on the speaker's bureau of Novartis, Bristol-Myers Squibb, Merck Sharp \& Dohme, Sanofi, Roche and Pierre Fabre. FG declares that has received consulting honoraria from Amgen, Merck Sharp \& Dohme, Bristol-Myers Squibb and Roche. GC declares that has received consulting honoraria, including travel and accommodation paid, from Janssen, IPSEN, Novartis, Roche, Pierre Fabre, BristolMyers Squibb, Sanofi, and EISAI, and is on the speaker's bureau of Roche, Merck, EVSA Pharma, EISAI, and Merck Sharp \& Dohme. JM declares that has received consulting honoraria, including travel and accommodation paid, from Roche, Bristol-Myers Squibb, and Novartis, and is on the speaker's bureau of Roche, Bristol-Myers Squibb, Novartis, Merck Sharp \& Dohme, and Pierre Fabre. MC declares that received consulting honoraria, including travel and accommodation paid, from Novartis, Roche, Pierre Fabre, and Merck Sharp \& Dohme. SO declares that has received consulting honoraria from Roche, Novartis, Merck Sharp \& Dohme, and BristolMyers Squibb. The authors report no other conflicts of interest in this work.

\section{References}

1. Guy GP Jr, Thomas CC, Thompson T, Watson M, Massetti GM, Richardson LC. Vital signs: melanoma incidence and mortality trends and projections - United States, 1982-2030. MMWR Morb Mortal Wkly Rep. 2015;64(21):591-596.

2. World Health Organization. Global cancer observatory. Melanoma of skin. Available from: http:/gco.iarc.fr/today/data/factsheets/cancers/ 16-Melanoma-of-skin-fact-sheet.pdf. Accessed June 16, 2020.

3. National Cancer Institute. Surveillance, epidemiology, and end results program. cancer stat facts: melanoma of skin. Available from: https:// seer.cancer.gov/statfacts/html/melan.html. Accessed June 16, 2020.

4. Cheng L, Lopez-Beltran A, Massari F, MacLennan GT, Montironi R. Molecular testing for BRAF mutations to inform melanoma treatment decisions: a move toward precision medicine. Mod Pathol. 2018;31 (1):24-38. doi:10.1038/modpathol.2017.104

5. Long GV, Menzies AM, Nagrial AM, et al. Prognostic and clinicopathologic associations of oncogenic BRAF in metastatic melanoma. $J \quad$ Clin Oncol. 2011;29(10):1239-1246. doi:10.1200/JCO.20 10.32.4327

6. Hugdahl E, Kalvenes MB, Puntervoll HE, Ladstein RG, Akslen LA. BRAF-V600E expression in primary nodular melanoma is associated with aggressive tumour features and reduced survival. $\mathrm{Br}$ J Cancer. 2016;114(7):801-808. doi:10.1038/bjc.2016.44

7. Chapman PB, Hauschild A, Robert C, et al. Improved survival with vemurafenib in melanoma with BRAF V600E mutation. $N$ Engl $J$ Med. 2011;364(26):2507-2516. doi:10.1056/NEJMoa1103782

8. Eroglu Z, Ribas A. Combination therapy with BRAF and MEK inhibitors for melanoma: latest evidence and place in therapy. Ther Adv Med Oncol. 2016;8(1):48-56. doi:10.1177/1758834015616934
9. Ascierto PA, Dreno B, Larkin J, et al. 5-year outcomes with cobimetinib plus vemurafenib in BRAFV600 mutation-positive advanced melanoma: extended follow-up of the coBRIM study. Clin Cancer Res. 2021. doi:10.1158/1078-0432.CCR-21-0809

10. Dréno B, Ascierto PA, Atkinson V, et al. Health-related quality of life impact of cobimetinib in combination with vemurafenib in patients with advanced or metastatic BRAFV600 mutation-positive melanoma. $\mathrm{Br} J$ Cancer. 2018;118(6):777-784. doi:10.1038/ bjc. 2017.488

11. Balch CM, Gershenwald JE, Soong SJ, et al. Final version of 2009 AJCC melanoma staging and classification. J Clin Oncol. 2009;27 (36):6199-6206. doi:10.1200/JCO.2009.23.4799

12. Knobel H, Alonso J, Casado JL, et al. Validation of a simplified medication adherence questionnaire in a large cohort of HIV-infected patients: the GEEMA Study. AIDS. 2002;16(4):605-613. doi:10.1097/00002030-200203080-00012

13. Reilly Associates. WPAI scoring. Available from: http://www.reillyas sociates.net/WPAI_Scoring.html. Accessed June 16, 2020.

14. Affleck AG, Stewart AM. The hospital anxiety and depression scale is a screening measure of general distress. Br J Dermatol. 2018;179 (2):544. doi:10.1111/bjd.16723

15. Ho AW, Tsao H. Targeted therapies in melanoma: translational research at its finest. J Invest Dermatol. 2015;135(8):1929-1933. doi:10.1038/jid.2015.14

16. Franken MG, Leeneman B, Gheorghe M, Uyl-de Groot CA, Haanen JBAG, van Baal PHM. A systematic literature review and network meta-analysis of effectiveness and safety outcomes in advanced melanoma. Eur J Cancer. 2019;123:58-71. doi:10.1016/j. ejca.2019.08.032

17. Di Guardo L, Cavalieri S, Cimminiello C, et al. Monoinstitutional experience of vemurafenib and cobimetinib for BRAF mutated metastatic melanoma. Ann Oncol. 2016;27(4):89-91. doi:10.1093/annonc/ mdw341.08

18. Drago JZ, Lawrence D, Livingstone E, et al. Clinical experience with combination BRAF/MEK inhibitors for melanoma with brain metastases: a real-life multicenter study. Melanoma Res. 2019;29(1):65-69. doi:10.1097/CMR.0000000000000527

19. Moser JC, Chen D, Hu-Lieskovan S, et al. Real-world survival of patients with advanced BRAF V600 mutated melanoma treated with front-line BRAF/MEK inhibitors, anti-PD-1 antibodies, or nivolumab/ipilimumab. Cancer Med. 2019;8(18):7637-7643. doi:10.1002/cam4.2625

20. Luke JJ, Ghate SR, Kish J, et al. Targeted agents or immuno-oncology therapies as first-line therapy for BRAF-mutated metastatic melanoma: a real-world study. Future Oncol. 2019;15 (25):2933-2942. doi:10.2217/fon-2018-0964

21. Cowey CL, Liu FX, Boyd M, Aguilar KM, Krepler C. Real-world treatment patterns and clinical outcomes among patients with advanced melanoma: a retrospective, community oncology-based cohort study (A STROBE-compliant article). Medicine. 2019;98 (28):e16328. doi:10.1097/MD.0000000000016328

22. Helgadottir H, Rocha Trocoli Drakensjö I, Girnita A. Personalized medicine in malignant melanoma: towards patient tailored treatment. Front Oncol. 2018;8:202. doi:10.3389/fonc.2018.00202

23. Arnold M, Jiang L, Stefanick ML, et al. Duration of adulthood overweight, obesity, and cancer risk in the women's health initiative: a longitudinal study from the United States. PLoS Med. 2016;13(8): e1002081. doi:10.1371/journal.pmed.1002081

24. Lennon H, Sperrin M, Badrick E, Renehan AG. The obesity paradox in cancer: a review. Curr Oncol Rep. 2016;18(9):56. doi:10.1007/ s11912-016-0539-4

25. McQuade JL, Daniel CR, Hess KR, et al. Association of body-mass index and outcomes in patients with metastatic melanoma treated with targeted therapy, immunotherapy, or chemotherapy: a retrospective, multicohort analysis. Lancet Oncol. 2018;19 (3):310-322. 
26. Calle EE, Rodriguez C, Walker-Thurmond K, Thun MJ. Overweight, obesity, and mortality from cancer in a prospectively studied cohort of U.S. adults. N Engl J Med. 2003;348(17):1625-1638. doi:10.1056/ NEJMoa021423

27. Fang S, Wang Y, Dang Y, et al. Association between body mass index, C-reactive protein levels, and melanoma patient outcomes. $J$ Invest Dermatol. 2017;137(8):1792-1795. doi:10.1016/j. jid.2017.04.007
28. Haugh AM, Johnson DB. Management of V600E and V600K BRAF-mutant melanoma. Curr Treat Options Oncol. 2019;20 (11):81. doi:10.1007/s11864-019-0680-z

\section{Publish your work in this journal}

OncoTargets and Therapy is an international, peer-reviewed, open access journal focusing on the pathological basis of all cancers, potential targets for therapy and treatment protocols employed to improve the management of cancer patients. The journal also focuses on the impact of management programs and new therapeutic

Submit your manuscript here: https://www.dovepress.com/oncotargets-and-therapy-journal agents and protocols on patient perspectives such as quality of life, adherence and satisfaction. The manuscript management system is completely online and includes a very quick and fair peer-review system, which is all easy to use. Visit http://www.dovepress.com/ testimonials.php to read real quotes from published authors. 